\title{
Pacific
}

Journal of

Mathematics

\section{JORDAN ANALOGS OF THE BURNSIDE AND JACOBSON DENSITY THEOREMS}

LUZIUS GRÜNENFELDER, M. OLMLADIČ AND HEYDAR RADJAVI 


\section{JORDAN ANALOGS OF THE BURNSIDE AND JACOBSON DENSITY THEOREMS}

\section{Grunenfelder, M. Olmladič and H. Radjavi}

If $\mathscr{A}$ is an (associative) algebra of linear operators on a vector space, it is well known that 2-transitivity for $\mathscr{A}$ implies density and, in certain situations, transitivity guarantees 2-transitivity. In this paper we consider analogs of these results for Jordan algebras of linear operators with the standard Jordan product.

0. Introduction. Let $\mathscr{L}(\mathscr{V})$ be the algebra of all linear operators on a vector space $\mathscr{V}$ over the field $\mathbb{F}$. A subset $\mathscr{S}$ of $\mathscr{L}(\mathscr{V})$ is called transitive if $\mathscr{S} x=\mathscr{V}$ for every nonzero $x$ in $\mathscr{V}$. More generally, $\mathscr{S}$ is called $k$-transitive if given linearly independent vectors $x_{1}, x_{2}, \ldots, x_{k}$ and arbitrary vectors $y_{1}, y_{2}, \ldots, y_{k}$ in $\mathscr{V}$ there exists a member $S$ of $\mathscr{S}$ such that $S x_{i}=y_{i}, i=1,2, \ldots, k$. If $\mathscr{S}$ is $k$-transitive for every $k$, then it is called (strictly) dense. It is a remarkable fact due to Jacobson [2] that if $\mathscr{S}$ is an (associative) subalgebra of $\mathscr{L}(\mathscr{V})$, then 2-transitivity implies density for arbitrary $\mathbb{F}$. In particular, if $\mathscr{V}$ is finite-dimensional, then $\mathscr{L}(\mathscr{V})$ is the only 2-transitive algebra on $\mathscr{V}$. There are transitive algebras that are not 2-transitive even if $\mathbb{F}$ is algebraically closed. In the presence of certain conditions (e.g., topological) transitivity implies density. The most well-known result of this kind is Burnside's theorem [3]: if $\mathscr{V}$ is finite-dimensional and $\mathbb{F}$ is algebraically closed, then the only transitive algebra over $\mathscr{V}$ is $\mathscr{L}(\mathscr{V})$.

In this paper we consider analogs of these results for Jordan algebras of operators: linear spaces $\mathscr{A}$ of operators such that $A^{2}$ and $A B A$ belong to $\mathscr{A}$ for all $A$ and $B$ in $\mathscr{A}$. If the characteristic of the field $\mathbb{F}$ is different from 2 , this is equivalent to the requirement that $\mathscr{A}$ be closed under the Jordan bracket $\{A, B\}=A B+B A$. Over this kind of field a Jordan algebra $\mathscr{A}$ may be equivalently defined as a linear space closed under taking positive integral powers. For the sake of completeness we include proofs of a few elementary facts obtainable from the general theory of Jordan algebras [4].

In what follows we often find it convenient to view members of $\mathscr{L}(\mathscr{V})$ as matrices over $\mathbb{F}$; this should cause no confusion. The set 
of all $n \times n$ matrices over $\mathbb{F}$ will be denoted by $\mathscr{M}_{n}(\mathbb{F})$. A member $A$ of $\mathscr{L}(\mathscr{V})$ (or $\left.\mathscr{M}_{n}(\mathbb{F})\right)$ is called a projection or an idempotent element if $A^{2}=A$.

\section{Transitive Jordan algebras over arbitrary fields.}

1.0. All the Jordan algebras $\mathscr{A}$ considered in this section are subalgebras of the algebra of all linear operators $\mathscr{L}(\mathscr{V})$ on a vector space $\mathscr{V}$ over a field $\mathbb{F}$. In finite dimensions Jacobson's theorem says that any 2-transitive associative algebra of linear operators of $\mathscr{V}$ is all of $\mathscr{L}(\mathscr{V})$ [2]. The proof of this result for Jordan algebras of operators needs some preparation.

1.1. Proposition. Let $\mathscr{A}$ be a Jordan algebra of linear operators on a vector space $\mathscr{V}$. Then:

(a) $E \mathscr{A} E$ and $(I-E) \mathscr{A}(I-E)$ are Jordan subalgebras of $\mathscr{A}$ for every $E$ in $\mathscr{A}$.

(b) If $\mathscr{V}$ is finite dimensional and $\mathscr{A}$ is 2-transitive, then for every subspace $\mathscr{W}$ of $\mathscr{V}$ there exists a projection $E \in \mathscr{A}$ such that $E \mathscr{V}=$ $\mathscr{W}$.

(c) If $\mathscr{V}$ is finite dimensional and $\mathscr{A}$ is 2-transitive, then $I \in \mathscr{A}$.

Proof. (a) follows directly from the definition and from the observation that $(I-E) \mathscr{A}(I-E)=A-E A-A E+E A E$.

(b) Assume first that $\mathscr{W}$ is a 1-dimensional subspace. By 2-transitivity there exists a singular $A \in \mathscr{A}$ such that $A \mathscr{W}=\mathscr{W}$. Choose $A$ to be of minimal rank having this property and write it in the form $A=J \oplus N$, where $J$ is invertible and $N$ is nilpotent. As all the powers of $A$ are in $\mathscr{A}$ and its rank is minimal, we have necessarily that $N=0$. The minimal polynomial $p(t)=\sum_{0 \leq i \leq m} a_{i} t^{i}$ of $J$ has nonzero constant term $a_{0}$ because $J$ is invertible. Thus, $I=$ $-\left(\sum_{1 \leq i \leq m} a_{i} J^{i}\right) / a_{0}$ is the identity operator on the range of $A$ and the idempotent $E=-\left(\sum_{1 \leq i \leq m} a_{i} A^{i}\right) / a_{0}=I \oplus 0$ is in $\mathscr{A}$. Moreover, $\operatorname{rank} E=\operatorname{rank} A$ and $E \mathscr{W}=\mathscr{W}$. If the rank of $E$ is strictly greater than 1, then let $E \mathscr{X}$ be a 1-dimensional subspace in the range of $E$ distinct from $E \mathscr{W}=\mathscr{W}$. By 2-transitivity there is a $B \in \mathscr{A}$ such that $B E \mathscr{W}=\mathscr{W}$ and $B E \mathscr{X}=0$. But then $E B E \mathscr{W}=\mathscr{W}$ and $E B E \mathscr{X}=0$ so that the rank of $E B E$, which is in $\mathscr{A}$ by part (a), is strictly smaller than the rank of $A$ contradicting the minimality assumption.

The rest follows by induction on the dimension of $\mathscr{W}$. Let $\mathscr{X}$ be a subspace of codimension 1 in $\mathscr{W}$ and $E \in \mathscr{A}$ a projection such 
that $E \mathscr{V}=\mathscr{X}$. Note that $\mathscr{Y}=\mathscr{W} \cap \operatorname{ker} E$ has dimension 1 and that $\mathscr{W}=\mathscr{X} \oplus \mathscr{Y}$. Let $F \in \mathscr{A}$ be a projection such that $F \mathscr{V}=\mathscr{Y}$; then $\mathscr{V}=\mathscr{W} \oplus \mathscr{U}$, where $\mathscr{U}=\operatorname{ker} E \cap \operatorname{ker} F$. Let $P$ be a projection in $\mathscr{V}$ on $\mathscr{W}$ along $\mathscr{U}$; then $N=E+F-P$ has square equal to zero and therefore, $P=2(E+F)-(E+F)^{2}$ is in $\mathscr{A}$. To get (c) take $\mathscr{W}=\mathscr{V}$ in (b).

Some of the proofs of the following results could be shortened slightly, at the expense of keeping the paper self-contained, by using the Pierce decomposition associated with an idempotent.

1.2. THEOREM. Let $\mathscr{A}$ be a Jordan algebra of linear operators on a finite dimensional vector space $\mathscr{V}$. Then $\mathscr{A}$ is 2-transitive if and only if $\mathscr{A}=\mathscr{L}(\mathscr{V})$.

Proof. $\mathscr{L}(\mathscr{V})$ is clearly 2 -transitive. The converse is proved by induction on the dimension of $\mathscr{V}$. The assertion obviously holds if $\operatorname{dim} \mathscr{V}=2$. So, let $\operatorname{dim} \mathscr{V}>2$. Let $\mathscr{X}$ be a 1-dimensional subspace of $\mathscr{V}$ and find by $1.1(\mathrm{~b})$ a projection $E \in \mathscr{A}$ such that $E \mathscr{V}=\mathscr{X}$. Next, find a 1-dimensional subspace $\mathscr{Y} \subset \operatorname{ker} E$ and corresponding projection $F \in \mathscr{A}$ such that $F \mathscr{V}=\mathscr{Y}$. It is clear that $E F=0$ and with no loss of generality we may assume that $F E=0$ as well, since otherwise, we could replace $F$ by $F-F E=$ $(I-E) F(I-E) \in \mathscr{A}$. The Jordan subalgebra $\mathscr{B}=(I-E) \mathscr{A}(I-E)$, respectively $\mathscr{C}=(I-F) \mathscr{A}(I-F)$, of $\mathscr{A}$ can be viewed as a 2transitive algebra of operators on $\operatorname{ker} E$, respectively $\operatorname{ker} F$, and by induction hypothesis $\mathscr{B}=\mathscr{L}(\operatorname{ker} \mathscr{E})$, respectively $\mathscr{C}=\mathscr{L}(\operatorname{ker} F)$. The subalgebra $\mathscr{C}$ is also called the Pierce zero-space relative to $F$. Choose now any $T \in \mathscr{L}(\mathscr{V})$ and let us show that $T \in \mathscr{A}$. By 2transitivity we may assume with no loss of generality that $E T F=$ $F T E=0$. But, then, $T=R+S$, where $R=(I-E) T(I-E) \in \mathscr{B}$ and $S=E T+T E-E T E \in \mathscr{C}$.

Theorem 1.2 can be generalized as follows for Jordan algebras of finite rank operators. For a further strengthening of this result see Theorem 3.4.

1.3. TheOREM. Let $\mathscr{A}$ be a Jordan algebra of finite rank operators on a vector space $\mathscr{V}$. If $\mathscr{A}$ is 2-transitive, then it is dense, i.e. $n$ transitive for all $n \geq 1$.

Proof. This can be done by reduction to the finite dimensional case. Observe that the proof of 1.1.(b) remains valid if we replace 
the condition " $\mathscr{V}$ is finite dimensional" by weaker conditions " $\mathscr{V}$ is finite dimensional and the elements of $\mathscr{A}$ have finite rank". Therefore, we can find for every finite dimensional subspace $\mathscr{W}$ of $\mathscr{V}$ a projection $E$ in $\mathscr{A}$ such that $E \mathscr{V}=\mathscr{W}$. By 1.1.(a) $E \mathscr{A} E$ is a Jordan subalgebra of $\mathscr{A}$ and it is 2-transitive as a Jordan algebra of operators on $E \mathscr{V}=\mathscr{W}$. Thus, $E \mathscr{A} E=\mathscr{L}(\mathscr{W})$ by 1.2. If vectors $\left\{x_{1}, x_{2}, \ldots, x_{k}\right\} \subset \mathscr{V}$ are linearly independent and vectors $\left\{y_{1}, y_{2}, \ldots, y_{k}\right\} \subset \mathscr{V}$ are arbitrary, then apply this consideration to the span $\mathscr{W}$ of these two sets of vectors.

\section{Some characterizations of proper transitive Jordan algebras.}

2.0. In this section we shall assume that the characteristic of the field $\mathbb{F}$ is different from 2 . Let $\mathscr{S}_{n}(\mathbb{F})$ be the (transitive) Jordan algebra of all symmetric $n \times n$ matrices over $\mathbb{F}$. We give a proof that if $\mathbb{F}$ is algebraically closed, then $\mathscr{S}_{n}(\mathbb{F})$ is, up to similarlity, the only proper transitive Jordan subalgebra of $\mathscr{M}_{n}(\mathbb{F})$. This, of course, does not hold if $\mathbb{F}$ is not algebraically closed. However, for a formally real closed field the algebra $\mathscr{S}_{n}(\mathbb{F})$ has no proper transitive Jordan subalgebras. These results do not seem to be easily derivable from Jacobson's general structure theorems for Jordan matrix algebras [4]; our presentation here is self-contained and elementary.

2.1. Theorem. Let $\mathbb{F}$ be any formally real closed field. Then, the only transitive Jordan algebra of symmetric $n \times n$ matrices over $\mathbb{F}$ is $\mathscr{S}_{n}(\mathbb{F})$.

Proof. We shall use induction on $n$. The assertion is trivial for $n=1$. So, assume $\mathscr{A}$ is a transitive Jordan subalgebra of $\mathscr{S}_{n}(\mathbb{F})$ with $n \geq 2$. Let $E$ be an idempotent of minimal positive rank in $\mathscr{A}$. Idempotents abound in $\mathscr{A}$ because in the spectral decomposition

$$
A=\sum \lambda_{i} E_{i}, \quad E_{i}^{2}=E_{i}, \quad E_{i} E_{j}=0, \quad i \neq j,
$$

for a member $A$ of $\mathscr{A}$, every $E_{i}$ corresponding to a nonzero $\lambda_{i}$ is a polynomial in $A$ (with constant term zero) and thus belongs to $\mathscr{A}$. The existence of spectral decompositions in $\mathscr{A}$ follows from the fact that $\mathbb{F}$ is real-closed [5].

The transitivity of $\mathscr{A}$ implies that it has nonscalar members, so that $E \neq I$. Since $E$ is symmetric there exists an invertible matrix $T$ with $T^{-1}=T^{t}$ such that

$$
T^{-1} E T=\left(\begin{array}{cc}
I_{k} & 0 \\
0 & 0
\end{array}\right)
$$


where $k$ is the rank of $E, 0<k<n$. Replacing $\mathscr{A}$ by $T^{-1} \mathscr{A} T$ we can assume that

$$
E=\left(\begin{array}{cc}
I_{k} & 0 \\
0 & 0
\end{array}\right)
$$

Writing the corresponding matrix for a typical member of $\mathscr{A}$

$$
A=\left(\begin{array}{cc}
X & Y^{\prime} \\
Y & Z
\end{array}\right)
$$

we observe that

$$
B=E A E-(I-E) A(I-E)=\left(\begin{array}{cc}
X & 0 \\
0 & -Z
\end{array}\right) \in \mathscr{A},
$$

and thus

$$
\frac{1}{2}(B E+E B)=\left(\begin{array}{cc}
X & 0 \\
0 & 0
\end{array}\right) \text { and }\left(\begin{array}{cc}
X & 0 \\
0 & 0
\end{array}\right)-B=\left(\begin{array}{ll}
0 & 0 \\
0 & Z
\end{array}\right)
$$

are in $\mathscr{A}$. By Proposition 1.1(a) we conclude that $E \mathscr{A} E$ and $(I-E) \mathscr{A}(I-E)$ are Jordan subalgebras of $\mathscr{A}$; they are also easily seen to be transitive on respective spaces $\operatorname{im} E$ and $\operatorname{ker} E$. Hence, by the inductive hypothesis, $E \mathscr{A} E=\mathscr{S}_{k}(\mathbb{F})$ and $(I-E) \mathscr{A}(I-E)=$ $\mathscr{S}_{n-k}(\mathbb{F})$. This means that $\mathscr{A}$ contains all symmetric matrices of the form $\left(\begin{array}{ll}L & 0 \\ 0 & M\end{array}\right)$. In particular, $k=1$ by minimality. To complete the proof observe that the transitivity of $\mathscr{A}$ forces it to contain a matrix with an arbitrarily assigned first column. Thus, for a given $(n-1) \times 1$ matrix $N$ there is a member $\left(\begin{array}{ll}L & N^{t} \\ N & M\end{array}\right)$ in $\mathscr{A}$ with some $L$ and $M$. Since by the argument above, $L=L^{t}$ and $M=M^{t}$ are arbitrary in this expression, we have that $\mathscr{A}=\mathscr{S}_{n}(\mathbb{F})$.

The following example shows that the hypothesis of real closure in the theorem is needed. Let $\mathbb{F}$ be the field $\mathbb{Q}$ of rational numbers and

$$
\mathscr{A}=\left\{\left(\begin{array}{cc}
a+b & b \\
b & a-b
\end{array}\right): a, b \in \mathbb{Q}\right\} .
$$

Then, $\mathscr{A}$ is a proper Jordan subalgebra of $\mathscr{S}_{2}(\mathbb{Q})$. It is easily seen that $\mathscr{A}$ is transitive: it is generated by $I$ and

$$
A=\left(\begin{array}{cc}
1 & 1 \\
1 & -1
\end{array}\right) \text {. }
$$

The minimal polynomial of $A$ is irreducible over $\mathbb{Q}$, and thus if $x$ is any nonzero vector, then the span of $x$ and $A x$ is the whole underlying space.

Our next theorem is a more general result in the case of algebraically closed fields; it includes, as a corollary, the analog of the above theorem. We need the following lemmas. 
2.2. LEMMA. Let $\mathscr{A}$ be a transitive Jordan algebra of $n \times n$ matrices over an algebraically closed field $\mathbb{F}$. Then $\mathscr{A}$ contains an idempotent of rank 1 .

Proof. For $n=1$ this is trivially true. We first prove it for $n=2$. Assume there is no idempotent of rank 1 in this case. This implies, by considering spectral projections of matrices in $\mathscr{A}$, that every member of $\mathscr{A}$ has singleton spectrum, i.e., it is of the form $N+\alpha I$ with $N$ nilpotent. Since the characteristic of $\mathbb{F}$ is different from 2, a member of $\mathscr{A}$ is nilpotent if and only if it has trace zero.

If $\mathscr{A}$ consists of nilpotents alone, then $0=(A+B)^{2}=A^{2}+$ $B^{2}+A B+B A=A B+B A$ for all $A, B \in \mathscr{A}$ implying that if $B \neq 0$, then its range is invariant under every $A \in \mathscr{A}$. Thus, $\mathscr{A}$ is triangularizable; this contradicts the transitivity of $\mathscr{A}$. Thus, we can assume that $\mathscr{A}$ has an invertible member, which implies, by taking an appropriate polynomial, that $I \in \mathscr{A}$. Hence, $N$ is in $\mathscr{A}$ for every $N+\alpha I$ in $\mathscr{A}$. Let $\mathscr{A}_{0}$ be the set of all nilpotent elements in the algebra. It follows that for $A$ and $B$ in $\mathscr{A}_{0}$ and $\alpha \in \mathbb{F}$, the matrix $A+\alpha B$ has trace zero and is thus nilpotent. In particular $A+B$ is nilpotent and hence $A B+B A=(A+B)^{2}-A^{2}-B^{2}=0$. This shows that $\mathscr{A}_{0}$ is a Jordan algebra. We see, as before, that $\mathscr{A}_{0}$ is triangularizable and so is $\mathscr{A}=\mathscr{A}_{0}+\mathbb{F} I$, contradicting the transitivity of $\mathscr{A}$.

We can now assume $n>2$. If $\mathscr{A}$ contains a nontrivial idempotent, i.e., an idempotent $E$ with $0<\operatorname{rank} E<n$, then by (1.1)(a) $E \mathscr{A} E$ is a Jordan subalgebra of $\mathscr{A}$ which is forced, by the transitivity of $\mathscr{A}$, to be transitive as an algebra of operators acting on the range of $E$. We conclude, by induction on $n$, that $E \mathscr{A} E$ and thus $\mathscr{A}$ contain idempotents of rank 1. To complete the proof we must only show the existence of a nontrivial $E$.

Assume $\mathscr{A}$ contains no nontrivial idempotent. Then, as in the first paragraph of the proof, every member of $\mathscr{A}$ is seen to be or the form $N+\alpha I$ with $N$ nilpotent. There must be nonzero nilpotent matrices in $\mathscr{A}$. (Just observe that if $N+\alpha I \in A$ with $\alpha \neq 0$, then, considering the characteristic polynomial of this matrix, we show that $I \in \mathscr{A}$ and hence $N \in \mathscr{A}$. Surely $\mathscr{A}$ cannot consist of scalar matrices.) Let $N$ be a nilpotent member of $\mathscr{A}$ with minimal positive rank. Since $N^{2}$ has smaller rank than $N$, we must have $N^{2}=0$. We next show that $N$ has rank 1. Considering members of $\mathscr{A}$ as operators on $\mathscr{V}$ and noting that the kernel of $N$ contains its range, let $\mathscr{V}_{1}$ be the 
range of $N, \mathscr{V}_{2}$ a complement of $\mathscr{V}_{1}$ in the kernel of $N$, and $\mathscr{V}_{3}$ a complement of $\mathscr{V}_{1} \oplus \mathscr{V}_{2}$ in $\mathscr{V}$. With respect to the decomposition $\mathscr{V}=\mathscr{V}_{1} \oplus \mathscr{V}_{2} \oplus \mathscr{V}_{3}$ (where $\mathscr{V}_{2}$ may of course be zero) and with an appropriate choice of basis, $N$ will have the form

$$
\left(\begin{array}{ccc}
0 & 0 & I_{k} \\
0 & 0 & 0 \\
0 & 0 & 0
\end{array}\right),
$$

where $k$ is the rank of $N$. If the corresponding block matrix of a typical $A$ in $\mathscr{A}$ is $\left(A_{i j}\right)_{i, j=1}^{3}$, then $N A N \in \mathscr{A}$ and its matrix equals

$$
\left(\begin{array}{ccc}
0 & 0 & A_{31} \\
0 & 0 & 0 \\
0 & 0 & 0
\end{array}\right) .
$$

The matrix $N A N-\alpha N$ is a nilpotent member of $\mathscr{A}$ and all its blocks except $A_{31}-\alpha I_{k}$ are zero. The minimality of the rank $k$ forces the block $A_{31}$ of every $A$ to be scalar. But this would contradict the transitivity of $\mathscr{A}$ if $k>1$. Thus, $k=1$. (These facts about algebras consisting of scalar translations of nilpotent operators can also be deduced from more sophisticated results on Jordan algebras [6].)

Finally, we shall exhibit a single member of $\mathscr{A}$ with an eigenvalue 1 , showing that not every member of $\mathscr{A}$ is the sum of a nilpotent and a scalar. To this end, pick $x \in \mathscr{V}$ with $N x \neq 0$. By transitivity, there is an $A \in \mathscr{A}$ such that $A(N x)=x$. Then, $N^{2}=0$ implies $(N A+A N)(N x)=N A N x=N x$. Since $N$ has rank 1, the matrix $N A+A N$ has rank at most 2. Since $n>2$, this matrix is a singular member of $\mathscr{A}$.

2.3. LemMA. If $\mathscr{A}$ satisfies the hypotheses of Lemma 2.2, then $\mathscr{A}$ contains idempotents $E_{i}, i=1,2, \ldots, n$, of rank one with $E_{i} E_{j}=0$ for $i \neq j$.

Proof. We shall use induction on $n$. Let $n \geq 2$ and assume the assertion true for $n-1$. Let $E_{1}=E$ be an idempotent of rank one in $\mathscr{A}$ as in Lemma 1.1. We can assume with no loss of generality that $E=\operatorname{diag}(1,0, \ldots, 0)$. The Jordan algebra $(I-E) \mathscr{A}(I-E)$ is contained in $\mathscr{A}$ by Proposition 1.1(a). Since it also acts transitively on the range of $I-E$, which has dimension $n-1$, we conclude from the inductive hypothesis that $(I-E) \mathscr{A}(I-E)$ contains idempotents $E_{2}, \ldots, E_{n}$ with the desired property. The proof is completed by observing that $E_{1} E_{j}=E_{j} E_{1}=0$ for $j \geq 2$. 
2.4. THEOREM. Let $\mathbb{F}$ be an algebraically closed field, and let $\mathscr{A}$ be a transitive Jordan algebra of $n \times n$ matrices over $\mathbb{F}$. Then either $\mathscr{A}=$ $\mathscr{M}_{n}(\mathbb{F})$ or there exists an invertible matrix $T$ such that $T^{-1} \mathscr{A} T=$ $\mathscr{S}_{n}(\mathbb{F})$.

Proof. We shall show first that $\mathscr{A}$ contains $\mathscr{S}_{n}(\mathbb{F})$ up to a similarity. By Lemma 2.3 we can assume that $\mathscr{A}$ contains diagonal idempotents $E_{j}$ of rank one: $E_{1}=\operatorname{diag}(1,0, \ldots, 0), \ldots, E_{n}=$ $\operatorname{diag}(0, \ldots, 0,1)$. Consider the special case of $n=2$. In this case the transitivity of $\mathscr{A}$ implies that its dimension is either 3 or 4 . If the dimension is 4 , then $\mathscr{A}=\mathscr{M}_{2}(\mathbb{F})$; if it is 3 , then $\mathscr{A}$ contains a nonzero matrix of the form $\left(\begin{array}{ll}0 & t \\ s & 0\end{array}\right)$ (after adding a suitable linear combination of $E_{1}$ and $E_{2}$ ). Now, both $s$ and $t$ have to be nonzero by transitivity. Thus, we have shown that, when $n=2$, the algebra $\mathscr{A}$ must contain a matrix of the above form with $s=1$ and $t \neq 0$.

Returning now to the general case, let $\left\{M_{i j}\right\}$ be the set of matrix units, i.e., the only nonzero entry of $M_{i j}$ occurs at the $(i, j)$ position and equals 1 . Observe that for $j>1$ the Jordan subalgebra $\left(E_{1}+E_{j}\right) \mathscr{A}\left(E_{1}+E_{j}\right)$ acts transitively on the 2-dimensional range of $E_{1}+E_{j}$. As in the paragraph above, it must contain, together with $E_{1}$ and $E_{j}$, at least one matrix of the form $A_{j}=M_{1 j}+t_{j} M_{j 1}$. Letting $T=\operatorname{diag}\left(1, \sqrt{t_{2}}, \ldots, \sqrt{t_{n}}\right)$, we see that the Jordan algebra $T \mathscr{A} T^{-1}$ contains the symmetric matrices

$$
B_{j 1}=\frac{1}{\sqrt{t_{j}}} T A_{j} T^{-1}=M_{j 1}+M_{1 j}
$$

(and, of course, $E_{1}, \ldots, E_{n}$ ).

If $1, i$, and $j$ are distinct, then $B_{i j}=B_{i 1} B_{j 1}+B_{j 1} B_{i 1} \in T \mathscr{A} T^{-1}$. Observe that $B_{i j}=M_{i j}+M_{j i}$. We have shown that $T \mathscr{A} T^{-1}$ contains a basis for symmetric matrices. Hence, $T \mathscr{A} T^{-1} \supset \mathscr{S}_{n}(\mathbb{F})$.

To complete the proof of the theorem it suffices to show that if $\mathscr{A}$ contains $\mathscr{S}_{n}(\mathbb{F})$ properly, then $\mathscr{A}=\mathscr{M}_{n}(\mathbb{F})$. Thus, assume $\mathscr{A}$ contains a nonsymmetric matrix $C=\left(c_{i j}\right)$. Some principal $2 \times 2$ submatrix must be nonsymmetric and by passing from $\mathscr{A}$ to $P^{-1} \mathscr{A} P$, where $P$ is a permutation matrix, we can assume $c_{12} \neq c_{21}$. Observe that the matrix

$$
M=\left(E_{1}+E_{2}\right) C\left(E_{1}+E_{2}\right)-c_{11} E_{1}-c_{22} E_{2}-c_{21} B_{21}
$$

belongs to $\mathscr{A}$ and is a nonzero scalar multiple of $M_{12}$. We shall show that $M_{i j} \in \mathscr{A}, i, j=1, \ldots, n$. Every $M_{i i}$ is in $\mathscr{A}$ and we have just seen that $M_{12}$ and hence $M_{21}=B_{21}-M_{12}$ are in $\mathscr{A}$. 
For $j>2, M_{1 j}=M_{12} B_{2 j}+B_{2 j} M_{12}$, and $M_{j 1}=B_{j 1}-M_{1 j}$ which implies that $M_{1 j}$ and $M_{j 1}$ are in $\mathscr{A}$. Similarly, for $j>i>1$, $M_{j i}=M_{1 i} B_{j 1}+B_{j 1} M_{1 i}$, and $M_{i j}=B_{i j}-M_{j i}$ so that $M_{j i}$ and $M_{i j}$ are in $\mathscr{A}$.

2.5. Corollary. Let $\mathbb{F}$ be an algebraically closed field. If $\mathscr{A}$ is a transitive Jordan algebra of symmetric $n \times n$ matrices over $\mathbb{F}$, then $\mathscr{A}=\mathscr{S}_{n}(\mathbb{F})$.

The example given after Theorem 2.1 can be modified to show that the algebraic closure hypothesis is essential in the preceding result. Consider $\mathbb{Q}(i)$ instead of $\mathbb{Q}$ and let

$$
\mathscr{A}=\left\{\left(\begin{array}{cc}
a+b & b \\
b & a-b
\end{array}\right): a, b \in \mathbb{Q}(i)\right\} .
$$

Then, $\mathscr{A}$ is a proper Jordan subalgebra of $\mathscr{S}_{2}(\mathbb{Q}(i))$; it is also transitive.

The following example shows that the assumption $\operatorname{char} \mathbb{F} \neq 2$ is essential in the results above: the 3-dimensional Jordan algebra spanned over $\mathbb{F}_{2}$ by $\left\{I, M_{12}, M_{21}\right\}$ is transitive and contains no idempotent of rank 1 .

\section{Results on ideals.}

3.0. We continue to assume that the characteristic of the field $\mathbb{F}$ is different from 2. In the associative algebra case some transitivity properties are inherited by ideals. This is of course trivial if $\operatorname{dim} \mathscr{V}$ is finite, since then $\mathscr{L}(\mathscr{V})$ is simple. In the Jordan case, restriction to ideals seems to be accompanied with some loss of transitivity. The following result is well known for general associative algebras [2].

3.1. Proposition. Let $\mathscr{J} \neq 0$ be an ideal in an associative algebra $\mathscr{A}$ of operators on a vector space $\mathscr{V}$. If $\mathscr{A}$ is $n$-transitive, then so is $\mathscr{J}$.

Here are our results on this question for Jordan algebras of linear operators and their Jordan ideals.

3.2. ThEOREM. Every Jordan ideal $\mathscr{J} \neq 0$ of an $(n+1)$-transitive Jordan algebra $\mathscr{A}$ of operators on a vector space $\mathscr{V}$ is $n$-transitive, $n \geq 1$.

Proof. If $\mathscr{V}$ is $(n+1)$-dimensional then $\mathscr{A}=\mathscr{L}(\mathscr{V})$ and, by [1, Theorem 1], we have $\mathscr{J}=\mathscr{A}$. So, assume with no loss of generality 
that $\mathscr{V}$ contains $n+2$ linearly independent vectors. Fix a linearly independent set of vectors $\left\{x_{1}, \ldots, x_{n}\right\} \subset \mathscr{V}$ and let $\mathscr{X}$ and $\mathscr{Y}$ be the span of $\left\{x_{1}, \ldots, x_{n}\right\}$ and of $\left\{x_{1}, \ldots, x_{n-1}\right\}$, respectively. We will show first that

(a) $\exists J \in \mathscr{J}$ such that $J \mathscr{Y}=0, J x_{n} \notin \mathscr{X}$. Assume the contrary; then

(b) $J \in \mathscr{J}$ and $J \mathscr{Y}=0$ implies $J x_{n} \in \mathscr{X}$.

Then, for any $A \in \mathscr{A}$ such that $A \mathscr{Y}=0$ it holds that $K=J A+A J$ is in $\mathscr{J}$ and that $K \mathscr{Y}=0$. Therefore by (b), $K x_{n}$ belongs to $\mathscr{X}$. Let $\alpha$ be such that $K x_{n}=(J+\alpha) A x_{n}$. Since $\mathscr{A}$ is $n$-transitive, $A$ may be chosen so that it satisfies the required conditions and that $A x_{n}$ is an arbitrary vector in $\mathscr{V}$. This shows that

(c) $J \mathscr{Y}=0$ implies $(J+\alpha) \mathscr{V} \subseteq \mathscr{X}$ for some $\alpha$. Thus,

$$
J=-\alpha+\sum_{1 \leq i \leq n} x_{i} \otimes f_{i}
$$

for some linear functionals $f_{i}, i=1, \ldots, n$. But then, for an arbitrary $A \in \mathscr{A}$ with $A \mathscr{Y}=0$ define $K$ as above and use the expression for $J$ to get

$$
K=-2 \alpha A+A x_{n} \otimes f_{n}+\sum_{1 \leq i \leq n} x_{i} \otimes f_{i} A
$$

Thus $K$ belongs to $\mathscr{J}$ and $K \mathscr{Y}=0$. Hence, by (c), it must be of the same form as $J$, i.e.,

$$
K=-\beta+\sum_{1 \leq i \leq n} x_{i} \otimes g_{i}
$$

Now, we choose vectors $u, v \in \mathscr{V}$ such that $\left\{x_{1}, \ldots, x_{n}, u, v\right\}$ are linearly independent, and find an $A \in \mathscr{A}$ such that $A \mathscr{Y}=0$, and that $A x_{n}=u, A u=v$. Then

$$
K u=-2 \alpha v+f_{n}(u) u+\sum_{1 \leq i \leq n} f_{i}(v) x_{i}=-\beta u+\sum_{1 \leq i \leq n} g_{i}(u) x_{i},
$$

which forces $\alpha=0$. A similar argument with $K$ playing the role of $J$ shows that $\beta=0$. Also, using the freedom in the choice of $u$, we conclude that $f_{n}$ is trivial. Thus, from the fact that (b) holds for every $J \in \mathscr{J}$ we obtain

(d) $J \in \mathscr{J}$ and $J \mathscr{Y}=0$ implies $J \mathscr{V} \subset \mathscr{Y}$.

The conclusion (d) contradicts the assumption that $\mathscr{J} \neq 0$ in case $n=1$. In other words, we have shown that given $x \neq 0$, there is a $J \in \mathscr{J}$ such that $x$ and $J x$ are linearly independent. Observe that this proves the theorem for $n=1$ : if $x \neq 0$ and $y$ are given 
and $J \in \mathscr{J}$ is such that $x$ and $J x$ are linearly independent, then by 2-transitivity choose an $A \in \mathscr{A}$ with $A x=x$ and $A J x=y-J x$. Then $A J+J A \in \mathscr{J}$ and $(A J+J A) x=y$.

Assume now for $n>1$ inductively that $\mathscr{J}$ is $(n-1)$-transitive and find an $E \in \mathscr{J}$ such that $E x_{i}=x_{i}, i=1, \ldots, n-1$. As $E^{2} \in \mathscr{J}$ and equals $E$ on $\mathscr{Y}$, we have by (d) that $\left(E-E^{2}\right) \mathscr{V} \subset \mathscr{Y}$. Assume now that there exists a vector $u \in \mathscr{V}$ such that $u$ and $E u$ do not belong to $\mathscr{Y}$. It follows from $\left(E-E^{2}\right) \mathscr{V} \subset \mathscr{Y}$ that $E^{2} u$ equals the sum of $E u$ and a vector from $\mathscr{Y}$ (so $E^{2} u \neq \mathscr{Y}$ ). By $n$-transitivity of $\mathscr{A}$ find an $A \in \mathscr{A}$ such that $A x_{i}=x_{i}, i=1, \ldots, n-1$, and $A E u=0$. This implies for $K=E A+A E \in \mathscr{J}$ that $K x_{i}=2 x_{i}, i=1, \ldots, n-1$ and $K E u \in \mathscr{Y}$. Hence, $K-2 E$ annihilates $\mathscr{Y}$ and its image is not contained in $\mathscr{Y}$, because $(K-2 E) E u$ equals the sum of $-2 E^{2} u$ and a vector from $\mathscr{Y}$, contradicting (d). The freedom in the choice of $u$ shows that for every $E \in \mathscr{J}$ such that $E x_{i}=x_{i}, i=1, \ldots, n-1$, we have necessarily that $E \mathscr{V} \subset \mathscr{Y}$ and $E$ is a projection on $\mathscr{Y}$. Choose now a nonzero vector $u \in \operatorname{ker} E$ and find by $(n+1)$-transitivity of $\mathscr{A}$ an $A \in \mathscr{A}$ such that $A x_{1}=u, A x_{i}=0, i=2, \ldots, n-1$, and $A u=x_{1}$. Then, $K=E A+A E-2 E A E \in \mathscr{J}$, and $K x_{1}=u, K x_{i}=0$, $i=2, \ldots, n-1$, and $K u=x_{1}$. It follows for $L=K^{2}-E K^{2} E \in \mathscr{J}$ that $L \mathscr{Y}=0$ and $L u=u$ contradicting (d). Consequently, we have shown that (b) leads to a contradiction and we have (a). So, fix a $J \in \mathscr{J}$ such that $J \mathscr{Y}=0$ and $J x_{n} \notin \mathscr{X}$. Now, pick by $(n+1)$ transitivity of $\mathscr{A}$ an $A \in \mathscr{A}$ such that $A \mathscr{X}=0$, and $A J x_{n}=u$ an arbitrary vector from $\mathscr{V}$. Thus, for $K=A J+J A \in \mathscr{J}$ we have that $K \mathscr{Y}=0$, and $K x_{n}=J A x_{n}+A J x_{n}=u$. The $n$-transitivity of $\mathscr{J}$ in the theorem now follows easily by cyclicly permuting the vectors $x_{i}$, $i=1, \ldots, n$ and taking sums of corresponding operators $K$.

The following result can also be obtained from work of Osborn and Racine [7].

3.3. COROllary. Every Jordan ideal of a dense Jordan algebra is dense.

3.4. THEOREM. Let $\mathscr{A}$ be a 2-transitive Jordan algebra of operators on a vector space $\mathscr{V}$. If $\mathscr{A}$ contains at least one operator of finite rank, then the Jordan ideal $\mathscr{J}$ of all finite rank operators of $\mathscr{A}$ is strictly dense, and so is $\mathscr{A}$.

Proof. Assume with no loss of generality that $\mathscr{V}$ is not finite dimensional. By $3.2 \mathscr{J}$ is transitive. Thus, we may find an $E \in \mathscr{J}$ 
such that $E x=x$ and such that it is of minimal rank with this property. Similarly as in the proof of 1.1.(b), we may find that $E$ is a projection of rank one and that for every finite dimensional subspace $\mathscr{W}$ of $\mathscr{V}$ we may find a projection $E \in \mathscr{J}$ such that $E \mathscr{V}=\mathscr{W}$. Now, for any $\{x, y\}$ linearly independent and $\{u, v\}$ arbitrary vectors of $\mathscr{V}$, let $\mathscr{W}$ denote the linear span of these four vectors and let $E \in \mathscr{J}$ be the corresponding projection. Use 2-transitivity of $\mathscr{A}$ to find $A \in \mathscr{A}$ such that $A x=u$ and $A y=v$, use 1.1.(a) to see that $B=E A E \in \mathscr{J}$, and observe that again $B x=u$ and $B y=v$. Thus, $\mathscr{J}$ is 2 -transitive and it is strictly dense by 1.3 .

The reader will no doubt have noticed that we left the following questions unanswered.

Question 1 . Is there an $n$-transitive Jordan algebra $\mathscr{A}$ with a Jordan ideal $\mathscr{J} \neq 0$ which is not $n$-transitive?

Question 2. Is there an $n$-transitive Jordan algebra which is not $(n+1)$-transitive for any $n \geq 2$ ?

\section{REFERENCES}

[1] I. N. Herstein, On the Lie and Jordan rings of a simple associative ring, Amer. J. Math., 77 (1955), 279-285.

[2] N. Jacobson, Structure of rings, Amer. Math. Soc. Colloq. Publ., vol. 37, Amer. Math. Soc., Providence, RI, 1984.

[3] _-, Lectures in Abstract Algebra, vol. II, Van Nostrand, (1953).

[4] - Structure and representations of Jordan algebras, Amer. Math. Soc. Colloq. Publ., vol. 39, Amer. Math. Soc., Providence, RI, 1968.

[5] S. Lang, Algebra, Addison Wesley, (1971).

[6] J. M. Osborn, Jordan and associative rings with nilpotent and invertible elements, J. Algebra, 15 (1970), 301-308.

[7] J. M. Osborn and M. L. Racine, Jordan rings with nonzero socle, Trans. Amer. Math. Soc., 251 (1979), 375-387.

Received December 15, 1991.

DALHOUSIE UNIVERSITY

HalifaX, Nova Scotia B3H 4H8

CANADA

AND

UNIVERSITY OF LJUBLJANA

61000 LJUBLJANA

SLOVENIA 


\title{
PACIFIC JOURNAL OF MATHEMATICS
}

Founded by

\author{
E. F. BECKenBaCH (1906-1982) F. WolF (1904-1989)
}

\section{EDITORS}

Sun-Yung A. Chang

(Managing Editor)

University of California

Los Angeles, CA 90024-1555

chang@math.ucla.edu

F. Michael Christ

University of California

Los Angeles, CA 90024-1555

christ@math.ucla.edu

Herbert Clemens

University of Utah

Salt Lake City, UT 84112

clemens@math.utah.edu

\author{
R. FInN
}

THOMAS ENRIGHT

University of California, San Diego

La Jolla, CA 92093

tenright@ucsd.edu

Nicholas ERColani

University of Arizona

Tucson, AZ 85721

ercolani@math.arizona.edu

Stanford University

Stanford, CA 94305

finn@gauss.stanford.edu

VAUghan F. R. JoNES

University of California

Berkeley, CA 94720

vfr@math.berkeley.edu

\section{SUPPORTING INSTITUTIONS}

STEVEN KeRCKHOFF

Stanford University

Stanford, CA 94305

spk@gauss.stanford.edu

MARTIN ScharLemanN

University of California

Santa Barbara, CA 93106

mgscharl@henri.ucsb.edu

HAROLd STARK

University of California, San Diego La Jolla, CA 92093

V. S. VARADARAJAN

University of California

Los Angeles, CA 90024-1555

vsv@math.ucla.edu

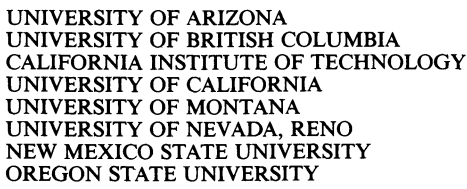

The Supporting Institutions listed above contribute to the cost of publication of this Journal, but they are not owners or publishers and have no responsibility for its content or policies.

Mathematical papers intended for publication in the Pacific Journal of Mathematics should be in typed form or offset-reproduced (not dittoed), double spaced with large margins. Please do not use built up fractions in the text of the manuscript. However, you may use them in the displayed equations. Underline Greek letters in red, German in green, and script in blue. The first paragraph must be capable of being used separately as a synopsis of the entire paper. In particular it should contain no bibliographic references. Please propose a heading for the odd numbered pages of less than 35 characters. Manuscripts, in triplicate, may be sent to any one of the editors. Please classify according to the 1991 Mathematics Subject Classification scheme which can be found in the December index volumes of Mathematical Reviews. Supply name and address of author to whom proofs should be sent. All other communications should be addressed to the managing editor, or Julie Honig, University of California, Los Angeles, California 90024-1555.

There are page-charges associated with articles appearing in the Pacific Journal of Mathematics. These charges are expected to be paid by the author's University, Government Agency or Company. If the author or authors do not have access to such Institutional support these charges are waived. Single authors will receive 75 free reprints; joint authors will receive a total of 100 free reprints. Additional copies may be obtained at cost in multiples of 50 .

The Pacific Journal of Mathematics (ISSN 0030-8730) is published monthly except for July and August. Regular subscription rate: $\$ 200.00$ a year (10 issues). Special rate: $\$ 100.00$ a year to individual members of supporting institutions.

Subscriptions, orders for numbers issued in the last three calendar years, and changes of address should be sent to Pacific Journal of Mathematics, P.O. Box 969, Carmel Valley, CA 93924, U.S.A. Old back numbers obtainable from Kraus Periodicals Co., Route 100, Millwood, NY 10546.

The Pacific Journal of Mathematics at P.O. Box 969, Carmel Valley, CA 93924 (ISSN 0030-8730) is published monthly except for July and August. Second-class postage paid at Carmel Valley, California 93924, and additional mailing offices. Postmaster: send address changes to Pacific Journal of Mathematics, P.O. Box 969, Carmel Valley, CA 93924.

PUBLISHED BY PACIFIC JOURNAL OF MATHEMATICS, A NON-PROFIT CORPORATION

This publication was typeset using $\mathcal{A} \mathcal{M S}-\mathrm{T}_{\mathrm{E}} \mathrm{X}$, the American Mathematical Society's $\mathrm{T}_{\mathrm{E}} \mathrm{X}$ macro system. Copyright (c) 1993 by Pacific Journal of Mathematics 


\section{PACIFIC JOURNAL OF MATHEMATICS}

Volume $161 \quad$ No. $2 \quad$ December 1993

On the method of constructing irreducible finite index subfactors of 201 Popa

FLORIN PETRE BOCA

Brownian motion and the heat semigroup on the path space of a

233 compact Lie group

JAY BARRY EPPERSON and TERRY M. LOHRENZ

Horizontal path spaces and Carnot-Carathéodory metrics

255

ZHONG GE

Biholomorphic convex mappings of ball in $\mathbb{C}^{n}$

SHENG GONG, SHI KUN WANG and QI HUANG YU

The Temperley-Lieb algebra at roots of unity

FREDERICK MiCHAEL GOODMAN and HANS WENZL

Jordan analogs of the Burnside and Jacobson density theorems

LUZIUS GRÜNENFELDER, M. OLMLADIČ and HEYDAR RADJAVI

Elliptic representations for $\mathrm{Sp}(2 n)$ and $\mathrm{SO}(n)$

REBECCA A. Herb

Reflexivity of subnormal operators

JOHN MCCARTHY

Knotting trivial knots and resulting knot types

KiMiHiko MOTEGI

Commutativity of selfadjoint operators

MitsURU UCHIYAMA

Correction to: "One-dimensional Nash groups" 